IMSc $/ 2002 / 12 / 41$

\title{
Topological field patterns of the Yang-Mills theory
}

\author{
E. Harikumar*, Indrajit Mitra ${ }^{\dagger}$ and H. S. Sharatchandra \\ The Institute of Mathematical Sciences, \\ C.I.T. Campus, Taramani P.O., \\ Chennai 600 113, India
}

\begin{abstract}
It is shown that the $S O(3)$ gauge field configurations can be completely characterised by certain gauge invariant vector fields. The singularities of these vector fields describe the topological aspects of the gauge field configurations. The topological (or monopole) charge is expressed in terms of an Abelian vector potential.
\end{abstract}

Keywords: Monopole, Abelianisation, Poincaré-Hopf Index.

PACS no: 14.80.Hv, 11.15.-q, 11.15.Tk

*hari@imsc.res.in

†indrajit@imsc.res.in

†sharat@imsc.res.in 


\section{Introduction}

In this letter we develop a formalism to describe generic topological field patterns of the Yang-Mills theory. This is done using certain gauge invariant special directions (actually orthonormal frames) [1] provided by the non-Abelian magnetic fields. These are the analogues of the Ricci principal directions [1] in general relativity. The singularities of these frames locate the topological aspects. We illustrate this explicitly in the case of the 't Hooft-Polyakov monopole [2]. This formalism provides a characterisation of the 't HooftPolyakov monopole using only the gauge fields, even in the interior. The framework is in the spirit of the Abelian projection procedure of 't Hooft [3]. The procedure has some connection with Ref. [4], but our emphasis is to use only the gauge fields and not the Higgs.

The topological character of the 't Hooft-Polyakov monopole [2] is mainly described by the behaviour of the Higgs field $\phi^{a}(x), a=1,2,3$, at spatial infinity: $\phi^{a}(x) \sim x^{a} / r$ for large

$r\left(=\sqrt{x^{a} x^{a}}\right)$. It has been recognized from the beginning [5] that such a behaviour of the Higgs field requires it to be zero at some point $x_{0}$ in the interior which may be identified with the 'centre' of the 't Hooft-Polyakov monopole. Using the Higgs field, we can construct the Poincaré -Hopf current [5]

$$
k_{i}=\frac{1}{2} \epsilon_{i j k} \epsilon_{a b c} \hat{\phi}^{a} \partial_{j} \hat{\phi}^{b} \partial_{k} \hat{\phi}^{c}
$$

where $\hat{\phi}^{a}=\phi^{a} /\left(\phi^{b} \phi^{b}\right)^{1 / 2}$ is the normalized Higgs field. This is divergenceless except at the centre $x_{0}$ where $\hat{\phi}^{a}$ is undefined:

$$
\partial_{i} k_{i}(x)=4 \pi \delta^{(3)}\left(x-x_{0}\right) .
$$

The monopole charge is thereby related to the topological charge,

$$
\begin{aligned}
M & =\frac{1}{4 \pi} \int d^{3} x \partial_{i} k_{i}(x) \\
& =\frac{1}{4 \pi} \oint_{S} d S^{i} k_{i}(x),
\end{aligned}
$$

where the surface integration can be carried over any surface $S$ enclosing the centre. This counts the number of times the normalized Higgs field covers the unit sphere in the isospace when we cover the surface $S$ once. In this way the Poincare-Hopf index of the isolated zero of the Higgs field gives the topological character of the 't Hooft-Polyakov monopole.

The 't Hooft-Polyakov monopole may also be described by gauge field using the 't Hooft Abelian magnetic field [2]

$$
b_{i}=\hat{\phi}^{a} B_{i}^{a}+\frac{1}{2} \epsilon_{i j k} \epsilon_{a b c} \hat{\phi}^{a} D_{j} \hat{\phi}^{b} D_{k} \hat{\phi}^{c}
$$

where $B_{i}^{a}=\epsilon_{i j k}\left(\partial_{j} A_{k}^{a}-\frac{1}{2} \epsilon_{a b c} A_{j}^{b} A_{k}^{c}\right)$ is the non-Abelian magnetic field and $D_{i} \hat{\phi}^{a}=\partial_{i} \hat{\phi}^{a}-$ $\epsilon_{a b c} A_{i}^{b} \hat{\phi}^{c}$ is the covariant derivative of the (normalized) Higgs field. We have

$$
b_{i}=\epsilon_{i j k} \partial_{j} a_{k}+k_{i}
$$

where $a_{i}=\hat{\phi}^{a} A_{i}^{a}$. The magnetic charge $\partial_{i} b_{i}$ comes entirely from the topological charge defined above: $\partial_{i} b_{i}(x)=4 \pi \delta^{(3)}\left(x-x_{0}\right)$. 


\section{Topological characterisation using only gauge fields}

It is to be noted that the above characterisation using the non-Abelian gauge potential, necessarily uses the Higgs field also. In the asymptotic region, $r \rightarrow \infty$, generalised Stokes' theorem can be used to characterise the monopole using only the non-Abelian gauge field $[6,7]$. But this approach does not work in the interior region. A topological characterisation of monopole using only gauge field everywhere including the interior has been realised recently [1].

A gauge invariant characterisation of monopole using only the non-Abelian gauge field is as follows: Consider a $3 \times 3$, real, symmetric matrix

$$
S_{i j}(x)=B_{i}^{a}(x) B_{j}^{a}(x)
$$

which is gauge invariant. Consider the eigenvalue equation

$$
S_{i j}(x) \zeta_{j}^{A}(x)=\lambda^{A}(x) \zeta_{i}^{A}(x), \quad A=1,2,3 .
$$

Here there is no summation over $A$. Since $S_{i j}$ is symmetric, the normalised eigenvectors $\zeta_{i}^{A}(x)$ provide an orthonormal frame at each $x$, i.e.,

$$
\zeta_{i}^{A}(x) \zeta_{i}^{B}(x)=\delta^{A B} .
$$

This frame is invariant under the local gauge transformations. The topological aspects of the monopole configuration can be related to the singularities of these three vector fields $\zeta_{i}^{A}(x), A=1,2,3$. To be specific, one of these, say $\zeta_{i}^{1}$, will appear to be diverging from the centre of the monopole. Thus here we have three orthonormal vectors to characterise the topological aspects completely in contrast with one unit vector used in the Faddeev-Niemi ansatz [8].

It is to be noted that the set $\lambda^{A}(x)$ and $\zeta_{i}^{A}(x)$ together provide the complete gauge invariant information about the non-Abelian gauge potential $A_{i}^{a}$. Clearly $A_{i}^{a}\left(\right.$ or $B_{i}^{a}$ ) have nine degrees of freedom at each $x$, of which three are gauge degrees. The six gauge invariant degrees can be now described by the three fields $\lambda^{A}(x)$ and the $3 \times 3$ orthogonal matrix $(\zeta(x))_{i A}=\zeta_{i}^{A}(x)$ (this matrix has three degrees of freedom, e.g., the Euler angles).

Instead of using the eigenvectors of $S_{i j}$ which is quadratic in non-Abelian magnetic field, we may construct the frame $\zeta_{i}^{A}$ as follows. The $3 \times 3$ matrix $(B)_{i a}=B_{i}^{a}$ can be made symmetric at each $x$ by an appropriate local gauge transformation. This is because any real matrix can be expressed as $B_{i}^{a}=(\mathcal{S} O)_{i a}$, where $\mathcal{S}$ is a real symmetric matrix ( not to be confused with $S_{i j}$ ) and $O$ is an orthogonal matrix. Here, $O$ can be removed by the local gauge transformation $B_{i}^{a} \rightarrow O_{a b} B_{i}^{b}$. In this special gauge, $S_{i j}=\left(B^{2}\right)_{i j}$, and so $\zeta^{\prime}$ s are the eigenfunctions of $B_{i}^{a}$.

Yet another way of constructing the frames $\zeta_{i}^{A}$ is as follows. By an appropriate local gauge transformation, we may make the three columns of the matrix $B_{i}^{a}$ mutually orthogonal (but not normalised). After normalisation, these columns give the frames $\zeta_{i}^{A}$. The reason is that any $B_{i}^{a}=\left(O_{1}^{T} \Lambda O_{2}\right)_{i a}$, where $O_{1}$ and $O_{2}$ are orthogonal matrices and $\Lambda$ is a diagonal matrix. By gauging away $O_{2}$, we get $B_{i}^{a}=\left(O_{1}^{T} \Lambda\right)_{i a}$ (which has mutually orthogonal columns). Substitution into (2.1) then shows that $O_{1} S O_{1}^{T}$ is diagonal, implying $\left(O_{1}^{T}\right)_{i A}=\zeta_{i}^{A}$. By normalizing the columns of $O_{1}^{T} \Lambda$, one obtains precisely this matrix $O_{1}^{T}$. 
For the subsequent analysis, we find it more useful to consider the symmetric tensor

$$
S^{a b}(x)=B_{i}^{a}(x) B_{i}^{b}(x),
$$

which is gauge covariant, instead of the gauge invariant tensor $S_{i j}$, and the normalised eigenfunctions $\xi_{a}^{A}$ :

$$
S^{a b}(x) \xi_{b}^{A}(x)=\lambda^{A}(x) \xi_{a}^{A}(x), \quad A=1,2,3 .
$$

It can readily checked that the eigenvalues are indeed the same for both the tensors, while the eigenfunctions are related: $B_{i}^{a} \xi_{a}^{A}$ is same as $\zeta_{i}^{A}$ up to a normalisation. (For Yang-Mills field configurations, generically the $3 \times 3$ matrix $B_{i}^{a}(x)$ is invertible [9].)

For each of $A=1,2$ and $3, \xi_{a}^{A}$ which is constructed from the non-Abelian gauge field, provides an isotriplet scalar, like the (normalised) Higgs field of the 't Hooft-Polyakov monopole. We will use these Higgs like fields to characterise the topological aspects of the non-Abelian gauge fields. We first illustrate this for 't Hooft-Polyakov monopole for which $S^{a b}$ has the general form

$$
S^{a b}=\alpha(r) \delta^{a b}+\beta(r) x^{a} x^{b}
$$

where $\alpha$ and $\beta$ are functions of only the radial distance $r$. One of the eigenvectors is the radial vector, $\xi_{a}^{1}=x^{a} / r$. The other two can be chosen to be any linearly independent combination of the basis vectors $\hat{\theta}$ and $\hat{\phi}$ of the spherical coordinate system, and these two are degenerate eigenfunctions of $S^{a b}$. This double degeneracy is a consequence of the spherical symmetry of the 't Hooft-Polyakov monopole solution.

It is important to note that the singularities in the eigenvector fields appear only at points where the eigenvalues become degenerate (because only at those points, the direction of an eigenvector can be indeterminate) [3]. For example, in the case of 't Hooft-Polyakov monopole, the eigenvalues are triply degenerate at the origin. Such a property is necessary because the entries of the matrix $B_{i}^{a}$ or $S^{a b}$ are themselves not singular at the origin. This provides a way to define centres of the monopoles (and other topological objects) for an arbitrary Yang-Mills field configuration.

\section{Abelian vector potential for Poincaré - Hopf current}

The eigenvector $\xi_{a}^{1}$ has unit topological charge at the origin. We construct the Poincaré -Hopf current for each of the three vectors $\xi_{a}^{A}$ :

$$
k_{i}^{A}=\frac{1}{2} \epsilon_{i j k} \epsilon^{a b c} \xi_{a}^{A} \partial_{j} \xi_{b}^{A} \partial_{k} \xi_{c}^{A},
$$

where there is no summation over $A$. Since $\partial_{i} k_{i}^{A}=0$ (except perhaps for Dirac delta function contribution due to the singularities of $\xi_{a}^{A}(x)$ ) we can express $k_{i}^{A}$ as a curl of a vector potential. We now obtain a formal expression for this vector potential. Regarding the orthogonal matrix $(\xi)_{a A}=\xi_{a}^{A}$ as a local gauge transformation, we get the corresponding pure gauge potential as

$$
\omega_{i}^{A}=\frac{1}{2} \epsilon^{A B C} \xi_{a}^{B} \partial_{i} \xi_{a}^{C} .
$$


Using this $\omega_{i}^{A}$, we re-express $k_{i}^{A}$ in terms of $\omega_{i}^{A}$ as

$$
\begin{aligned}
k_{i}^{A} & =\frac{1}{2} \epsilon_{i j k} \epsilon^{A B C} \xi_{b}^{B} \xi_{c}^{C} \partial_{j} \xi_{b}^{A} \partial_{k} \xi_{c}^{A} \quad \text { (no summation over A) } \\
& =\frac{1}{2} \epsilon_{i j k} \epsilon^{A B C} \omega_{j}^{B} \omega_{k}^{C},
\end{aligned}
$$

where we have used the fact $\operatorname{det} \xi_{a A}=1$ in the first step and $\epsilon^{A B C} \epsilon^{A B D} \epsilon^{A C E}=\epsilon^{A D E}$ (no sum over $A$ ) in the second. Since $\omega_{i}^{A}$ is a pure gauge, the corresponding non-Abelian magnetic field vanishes, i.e.,

$$
\epsilon_{i j k}\left(\partial_{j} \omega_{k}^{A}-\frac{1}{2} \epsilon^{A B C} \omega_{j}^{B} \omega_{k}^{C}\right)=0
$$

This allows us to write $k_{i}^{A}$ as a curl:

$$
k_{i}^{A}=\epsilon_{i j k} \partial_{j} \omega_{k}^{A}
$$

When monopole and other topological objects are present, some $\xi_{a}^{A}$ have singularities at the centres. Then, in general, $\partial_{i} k_{i}^{A}$ will have Dirac delta function singularities. For such a situation (3.5) is to be modified exactly in the same manner as Dirac's construction of the vector potential of a monopole:

$$
k_{i}^{A}=\epsilon_{i j k} \partial_{j} \omega_{k}^{A}-\text { Dirac string contributions . }
$$

In this case, $\omega_{i}^{A}$ is not strictly a pure gauge, and (3.4) gets modified to

$$
\epsilon_{i j k}\left(\partial_{j} \omega_{k}^{A}-\frac{1}{2} \epsilon^{A B C} \omega_{j}^{B} \omega_{k}^{C}\right)=\text { Dirac string contributions . }
$$

By this procedure, we have succeeded in describing the topological objects of the nonAbelian gauge fields in an Abelian fashion. The topological features are contained in the (ordinary) curl of a vector potential $\left(\omega_{i}^{A}\right)$ without requiring the non-linear terms. We will now illustrate these features in the case of 't Hooft-Polyakov monopole.

The Poincaré-Hopf currents $k_{i}^{A}$ for the 't Hooft-Polyakov monopole are as follows. We have already taken $\xi_{a}^{1}=\hat{x}^{a}$. Let us choose $\xi_{a}^{2}=\hat{\theta}^{a}$ and $\xi_{a}^{3}=\hat{\phi}^{a}$. On going over to spherical polar coordinates, (3.1) becomes

$$
k_{i}^{A}=\hat{x}_{i} \frac{1}{r^{2} \sin \theta} \epsilon^{a b c} \xi_{a}^{A} \frac{\partial \xi_{b}^{A}}{\partial \theta} \frac{\partial \xi_{c}^{A}}{\partial \phi}
$$

from which, for the 't Hooft-Polyakov monopole, we get

$$
\begin{aligned}
k_{i}^{1} & =\hat{x}_{i} \frac{1}{r^{2}}, \\
k_{i}^{2} & =\hat{x}_{i} \frac{\cot \theta}{r^{2}}, \\
k_{i}^{3} & =0 .
\end{aligned}
$$

Here we note that $k_{i}^{1}$ is precisely the magnetic field of a Dirac monopole. The flux over any surface enclosing the origin is then given by

$$
\oint d S^{i} k_{i}^{1}=4 \pi
$$


Note that the magnetic current corresponding to $A=2$, viz. $k_{i}^{2}$ is also non-zero. However, the corresponding magnetic charge is zero, i.e.,

$$
\oint d S^{i} k_{i}^{2}=0
$$

It corresponds to a radial flux from the region $z<0$ to the region $z>0$.

Using (3.2), we find the vector potentials $\omega_{i}^{A}$ for the 't Hooft-Polyakov monopole are

$$
\begin{array}{r}
\omega_{i}^{1}=-\hat{\phi}_{i} \frac{\cot \theta}{r}, \\
\omega_{i}^{2}=\hat{\phi}_{i} \frac{1}{r}, \quad \omega_{i}^{3}=-\hat{\theta}_{i} \frac{1}{r} .
\end{array}
$$

The potential $\omega_{i}^{1}$ is to be compared with (appropriately scaled, viz. $A \rightarrow e A$ ) Dirac potential for a monopole

$$
A_{i}(x)=\hat{\phi}_{i} \frac{e M}{4 \pi r} \frac{(1-\cos \theta)}{\sin \theta},
$$

where $e$ is the electric charge and $M$ is the magnetic charge. This has the Dirac string along the negative $z$-axis. Consider the average of the Dirac potentials with string along the positive $z$-axis and positive $z$-axis (obtained from (3.14) by changing $\theta \rightarrow \pi+\theta$ ). This gives the magnetic field of a monopole of Schwinger charge $M=4 \pi / e$ exactly same as the $\omega_{i}^{1}$ in (3.12).

It can be explicitly checked that, on ignoring the Dirac string contribution, $\epsilon_{i j k} \partial_{j} \omega_{k}^{A}$ gives precisely the same Poincaré -Hopf current $k_{i}^{A}$, and the same winding numbers. Thus, the vector potential for $A=2$ gives magnetic flux without monopole, while the vector potential for $A=3$ does not give magnetic flux. Their relevance is elucidated later.

\section{4 'Abelianisation' of Yang-Mills potential}

Consider a gauge transformation using the orthogonal matrix $(\xi)_{a A}=\xi_{a}^{A}$. The transformed potentials are

$$
\begin{aligned}
a_{i}^{A} & =\xi_{a}^{A} A_{i}^{a}+\frac{1}{2} \epsilon^{A B C} \xi_{a}^{B} \partial_{i} \xi_{a}^{C} \\
& =\tilde{A}_{i}^{A}+\omega_{i}^{A} .
\end{aligned}
$$

Here $\tilde{A}_{i}^{A}$ is the Abelian vector potential of 't Hooft now constructed using the three Higgs like fields $\xi_{a}^{A}$ ( compare $a_{i}$ given just after (1.5)). When monopoles are present, this gauge transformation is singular due to singularities of $\xi_{a}^{A}$ (see (3.7)). As an explicit example we consider the 't Hooft-Polyakov monopole [2]:

$$
A_{i}^{a}=-\epsilon_{a i j} \hat{x}^{j} \frac{1-K(r)}{r},
$$

where $K(r) \rightarrow 0$ as $r \rightarrow \infty$ and $K(r)=1+O\left(r^{2}\right)$ as $r \rightarrow 0$. So we get

$$
\begin{aligned}
\tilde{A}_{i}^{1} & =0, \\
\tilde{A}_{i}^{2} & =-\hat{\phi}_{i} \frac{1-K(r)}{r}, \\
\tilde{A}_{i}^{3} & =\hat{\theta}_{i} \frac{1-K(r)}{r} .
\end{aligned}
$$


Making use of (3.12) and (3.13), we then obtain

$$
\begin{aligned}
a_{i}^{1} & =-\hat{\phi}_{i} \frac{\cot \theta}{r}, \\
a_{i}^{2} & =\hat{\phi}_{i} \frac{K(r)}{r}, \\
a_{i}^{3} & =-\hat{\theta}_{i} \frac{K(r)}{r} .
\end{aligned}
$$

For $A=1$ we recover the Dirac potential of a point monopole $\left(a_{i}^{2}\right.$ and $a_{i}^{3}$ vanish asymptotically) while the orthogonal transformation provided by $\xi_{a}^{A}$ rotates $\hat{x}^{a}$ into 1 -direction. This is similar to the singular gauge transformation rotating the Higgs field $\phi^{a}$ to the 3 -direction in Ref. [5]. However, we obtained this transformation using only the gauge potential.

The relevance of $a_{i}^{2}$ and $a_{i}^{3}$ is the following. In an Abelian theory, the energy of the Dirac monopole would diverge due to the singularity of $a_{i}^{1}$ at the origin. Now however $a_{i}^{2}$ and $a_{i}^{3}$ also diverge in a specific way to ensure that the nonlinear terms $\epsilon_{i j k} a_{j}^{2} a_{k}^{3}$ in $B_{i}^{1}$ cancel the singular contribution of $a_{i}^{1}$.

The three new potentials $a_{i}^{A}$, when regarded as three Abelian vector potentials, carry all the information of the non-Abelian topology. For $A=1$ we have a Dirac monopole charge, while the $A=2,3$ cases have none.

Note that $a_{i}^{A}=\xi_{a}^{A}\left(A_{i}^{a}-\tilde{\omega}_{i}^{a}\right)$ where $\tilde{\omega}_{i}^{a}=\frac{1}{2} \epsilon_{a b c} \xi_{b}^{B} \partial_{i} \xi_{c}^{B}$. Both $A_{a}^{i}$ and $\tilde{\omega}_{i}^{a}$ transform inhomogeneously as gauge potentials under local gauge transformation. Therefore $A_{i}^{a}-\tilde{\omega}_{i}^{a}$ transforms homogeneously as a triplet, and its scalar product with $\xi_{a}^{A}$ is invariant. Thus $a_{i}^{A}$ are gauge invariant under the non-Abelian gauge transformation acting on the subscript $a$ in (4.1). The superscript $A$ provides gauge invariant directions as obtained from the eigenfunctions of $S_{i j}$. Instead of the non-Abelian Wilson loop $\operatorname{Tr} \mathcal{P} \exp \left(i \oint d x^{i} A_{i}\right)$, we can consider three Abelian Wilson loops

$$
W^{A}[C]=\exp \left(i \oint_{C} d x^{i} a_{i}^{A}\right) .
$$

These are gauge invariant (under the non-Abelian gauge transformation) and carry all topological information about the non-Abelian gauge fields.

\section{Conclusion}

In this letter, we have shown that the non-Abelian $(S O(3))$ gauge field configurations can be completely characterised by certain gauge invariant vector fields. The singularities of these vector fields describe the topological aspects of the gauge field configurations. Our procedure provides an Abelianisation of the non-Abelian gauge theory in two ways:

1. The topological (or monopole) charge is characterised by the curl of an Abelian vector potential.

2. The non-Abelian gauge field is transformed to three 'gauge invariant' vector potentials and they capture the topological aspects when treated as Abelian vector potentials. 
Using this approach, we can obtain the most general topological field patterns of the YangMills fields. For example, one finds that the generic configuration has half the 't HooftPolyakov monopole charge. Also there are vortices of half-integral winding number. These aspects will be elaborated elsewhere [10].

\section{References}

[1] R. Anishetty, P. Majumdar and H. S. Sharatchandra, Phys. Lett. B478 (2000) 373.

[2] G. 't Hooft, Nucl. Phys. B79 (1974) 276; A. M. Polyakov, JETP Lett. 20 (1974) 194.

[3] G. 't Hooft, Nucl. Phys. B190 (1981) 455.

[4] A. Di Giacomo and M. Mathur, Phys. Lett. B400 (1997) 129.

[5] J. Arafune, P. G. O. Freund and C. J. Goebel, J. Math. Phys. 16 (1975) 433.

[6] P. Goddard and D. Olive, Rep. Prog. Phys. 41 (1978) 1357.

[7] See Sec. 5.8 of Ref. [6] and references therein.

[8] L. Faddeev and A. J. Niemi, Phys. Rev. Lett. 82 (1999) 1624.

[9] P. Majumdar and H. S. Sharatchandra, Phys. Rev. D63 (2001) 067701.

[10] E. Harikumar, Indrajit Mitra and H. S. Sharatchandra, 'Half-monopoles and halfvortices in the Yang-Mills theory', IMSc preprint, IMSc/2002/12/42. 